\title{
СРАВНИТЕЛЬНЫЙ АНАЛИЗ МЕТОДОВ СЕМАНТИЧЕСКОГО МОДЕЛИРОВАНИЯ: ЕRА-МОДЕЛЬ ЧЕНА И МЕТОД КОНЦЕПТУАЛЬНОГО МОДЕЛИРОВАНИЯ В МЕТОДОЛОГИИ АВТОМАТИЗАЦИИ ИНТЕЛЛЕКТУАЛЬНОГО ТРУДА (МАИТ)
}

\section{COMPARATIVE ANALYSIS OF SEMANTIC MODELING METHODS: CHEN'S ERA MODEL AND THE CONCEPTUAL MODELING METHOD \\ IN THE METHODOLOGY OF AUTOMATION OF INTELLECTUAL WORK (MAIW)}

G. Volkova Nyi Nyi Htwe

Summary. The purpose of this work is to analyze existing approaches in the field of conceptual (semantic) modeling of subject problems.

Keywords: Chen method, conceptual modeling method, semantic modeling of subject problems, methodology of automation of intellectual work (MAIW).

\section{Ввехение}

A нализ эволюции технологий программирования в разных подходах - традиционном, структурном, объектно-ориентированном, а также в логическом и функциональном программировании - позволил установить, что доминирующим методологическим базисом при моделировании семантики предметной области является методология Чена. Новый подход когнитивный, принципиально отличается от вышеперечисленных технологических парадигм тем, что моделирование семантики выполняется с учетом уровней семантической сложности и закона цикличности, обеспечивающего увязку уровней. В настоящее время очень мало разработано методов моделирования в рамках этого подхода. Одним из таких методов является метод концептуального моделирования в рамках методологии автоматизации интеллектуального труда, соответствующей когнитивному подходу.

Для того, чтобы сравнивать эти методы, необходимо выбрать критерии. В качестве критериев были выбраны следующие:

\author{
Волкова Галина Дмитриевна \\ Д.т.н., nрофессор, МГТУ «СТАНКИН», г. Москва \\ cog-par@yandex.ru \\ Hou Hou Xmse \\ Аспирант, МГТУ «СТАНКИН», г. Москва \\ sawnyinyi90@yandex.ru
}

Аннотация. Целью работы является анализ существующих подходов в области концептуального (семантического) моделирования предметных задач.

Ключевые слова: метод Чена, метод концептуального моделирования, семантическое моделирование предметных задач, методология автоматизации интеллектуального труда (МАИТ).

- место семантического моделирования при автоматизации информационных и интеллектуальных задач;

- методологические основания для семантического моделирования;

- наличие и разнообразие семантических модельных представлений;

- наличие уровней абстрагирования в модельных представлениях;

- наличие составляющих в семантических моделях

- наличие формального аппарата для семантических моделей;

- наличие закономерностей формирования семантических моделей.

С учетом выделенных критериев рассмотрим каждый метод.

\section{Метолология Чена}

В традиционном программировании информационное представление определялось возможностями вычислительной техники - обработкой числовых и сим- 
вольных данных [1,2,3]. Исходя из этого, использовалось упрощенное представление о реальном мире, как о наборе или множестве характеристик реальных объектов.

Содержательно эти характеристики представляли количественные и/или качественные атрибуты объектов и в компьютере описывались множеством переменных.

Формальной моделью информационного представления в ЭВМ являлся аппарат теории формальных грамматик. Автоматизация все более сложных расчетных задач привела к следующим проблемам:

- большое количество переменных задачи усложняло и написание программ, и их отладку, и тестирование;

- дублирование данных в комплексных задачах;

- сложность извлечения данных из ЭВМ (в виде программ, реализующих запросы) и др.

Переход к структурному программированию характеризовался качественным скачком, а именно: переход от множества переменных

- данных к структурам на них. Это повлекло изменение идеи и в семантических представлениях. Реальный мир стал отражаться через объекты и их связи.

Моделирование данных в виде структур данных получило формальные воплощения в вычислительной среде, наиболее полным из которых явилась реляционная модель Кодда. Методологическое обоснование новой технологии программирования было предложено Ченом в начале 70-х годов XX века и получило название подхода «сущность-связь-атрибут», или ERA-подхода. Этот подход занимает доминирующую методологическую позицию и в настоящее время.

Модель «сущность-связь» основывается на существенной информации о реальном мире и предназначена для описания на мета-уровне. С ее помощью осуществляется детализация структур данных проектируемой системы, включая идентификацию важных для предметной области объектов (сущностей -Entity), их свойств (атрибутов -Attribute) и отношений с другими объектами (связей-Relationship).

Семантическое моделирование предметной области выполняется перед проектированием информационных систем или баз данных.

В методологии Чена главный упор делается на семантическое моделирование статической составляющей предметной области, при этом само моделирование осуществляется в визуальной форме - форме специальных диаграмм.
Особенностью методологии Чена является отображение семантических элементов и конструкций в синтаксические - реляционные отношения Кодда. Так и сущности, и связи отображаются в реляционные отношения в виде множеств атрибутов-доменов. При этом в методологии отсутствует какой-либо формальный аппарат и формирование сложных семантических структур возлагается на специалистов-аналитиков, их опыт и знания.

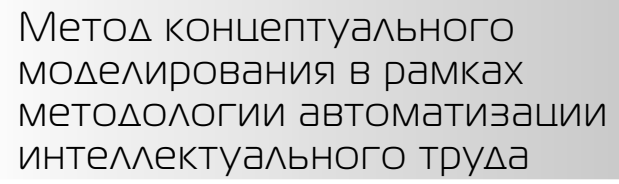

Методология автоматизации интеллектуального труда разрабатывалась для обеспечения промышленного способа создания автоматизированных информационных и интеллектуальных систем [4,5]. Особенностью методологии является последовательное отображение формально-языковых представлений на основе первоначально формируемого концептуального (семантического) представления.

Для концептуального моделирования были выделены следующие методологические основания:

- методология создания машин по Ю.М. Соломенцеву;

- категории и законы диалектики;

- структура процесса познания;

- анализ философского понятия «производство».

Концептуальное моделирование в рамках методологии рассматривается на трех уровнях абстрагирования и для двух представлений: универсального концептуального представления и концептуальных представлений предметных задач. Универсальное концептуальное представление (УКП) определяет общее строение системы знаний на трех уровнях абстрагирования, а концептуальное представление предметных задач (КППЗ) определяют строение знаний с учетом специфики предметной области на двух уровнях абстрагирования: одна концептуальная модель объектного уровня и множество концептуальных моделей конкретного уровня. При этом концептуальная модель объектного уровня описывает предметные знания в различных методиках решения предметных задач, а концептуальная модель конкретного уровня описывает знания в виде конкретных решений.

Каждая концептуальная модель представляет собой совокупность взаимосвязанных конструкций: статических, динамических и их увязку. Для описания концептуальных представлений, моделей и их составляющих был разработан формальный аппарат, который позволяет представлять элементы и связи конструкций для моде- 
Таблица 1. Сравнительный анализ двух методов

\begin{tabular}{|l|l|l|}
\hline Критерии сравнения & Методология Чена & $\begin{array}{l}\text { Метод концептуального моделирования в рамках } \\
\text { методологии автоматизации интеллектуального } \\
\text { труда }\end{array}$ \\
\hline Наличие методологических оснований & нет & есть \\
\hline $\begin{array}{l}\text { Наличие и разнообразие семантических } \\
\text { модельных представлений }\end{array}$ & нет & есть (универсальное и предметное) \\
\hline $\begin{array}{l}\text { Наличие уровней абстрагирования в модельных } \\
\text { представлениях }\end{array}$ & мета-уровень & $\begin{array}{l}\text { три уровня абстрагирования -абстрактный, } \\
\text { объектный, конкретный }\end{array}$ \\
\hline $\begin{array}{l}\text { Наличие составляющих в семантических } \\
\text { моделях }\end{array}$ & $\begin{array}{l}\text { только статическая } \\
\text { составляющая }\end{array}$ & статическая, динамическая, их увязка \\
\hline $\begin{array}{l}\text { Наличие формального аппарата для } \\
\text { семантических моделей }\end{array}$ & нет & \begin{tabular}{l} 
оригинальный формальный аппарат \\
\hline $\begin{array}{l}\text { Наличие закономерностей формирования } \\
\text { семантических моделей }\end{array}$ \\
концептуальных моделей и их увязки в единое целое \\
в универсальном представлении
\end{tabular} \\
\hline
\end{tabular}

лей разных уровней абстрагирования, но и формально описывать связи моделей в рамках каждого представления. В качестве элементов статических отношений используются понятия-категории с учетом уровней абстрагирования, на которых устанавливаются бинарные и тернарные связи.

Тернарные связи позволили сформировать сложные производные элементы - схемы категорий, на которых также устанавливаются бинарные связи. В качестве элементов динамических отношений используются ограничения - зависимости 1-го и 2-го рода. Система ограничений 1-го рода увязана с основной концептуальной структурой, а система ограничений 2-го рода - с производной концептуальной структурой в рамках модели любого уровня абстрагирования и любого представления.

Формально описаны связи структур разных концептуальных представлений. Отличительной особенностью метода концептуального моделирования в рамках методологии является наличие закономерностей формиро- вания концептуальных моделей в универсальном представлении как проявление закона диалектики (закона отрицания отрицания).

\section{Сравните^ьный анализ $\triangle$ вух по $А$ поОв к моАелированию семантики}

На основании изложенного можно выполнить сравнительный анализ двух методов семантического моделирования предметных областей и/или задач. Результаты сравнительного анализа приведены в табл. 1.

\section{Зак^ючение}

Метод концептуального моделирования в рамках методологии автоматизации интеллектуального труда ориентирован на когнитивный подход к моделированию знаний (семантики) предметных задач, обеспечивающий многоуровневое масштабирование семантических представлений этих задач и, таким образом, метод Чена является частным случаем метода концептуального моделирования.

\section{ЛИТЕРАТУРА}

1. Кара-Ушанов В. Ю. Модель «Сущность — Связь»: учеб. пособие / В. Ю. Кара-Ушанов. — Екб.: — Электронное текстовое издание, 2017. — 64 с.

2. Пинягина 0. В. Лекции «Базы Данных»: ER-модель (entity-relationship) [Электронный ресурc]. URL: http://kek.ksu.ru/EOS/BD/ER_model.html (дата oбpaщения: 02.03.2019)

3. Модель «Сущность — Связь» [Электронный ресурс].— Режим доступа: https://en.wikipedia.org/wiki/Entity- relationship_model (дата обращения: 06.04.2019)

4. Волкова Г. Д. Концептуальное моделирование проектных задач: учеб. пособие / Г. Д. Волкова.— М.: ФГБОУ ВО «МГТУ «СТАНКИН», 2015.

5. Волкова Г. Д. Методология автоматизации интеллектуального труда. Г. Д. Волкова. — М.: Янус-К, 2013.— 102 с. 